\title{
Stress-Softening and Residual Strain Effects in Suture Materials
}

\author{
Alex Elías-Zúñiga, ${ }^{1}$ Beatriz Montoya, ${ }^{1}$ Wendy Ortega-Lara, ${ }^{1}$ Eduardo Flores-Villalba, ${ }^{1,2}$ \\ Ciro A. Rodríguez, ${ }^{1}$ Hector R. Siller, ${ }^{1}$ José A. Díaz-Elizondo, ${ }^{2}$ and Oscar Martínez-Romero ${ }^{1}$ \\ ${ }^{1}$ Centro de Innovación en Diseño y Tecnología, Tecnológico de Monterrey-Campus Monterrey, \\ Avenida E. Garza Sada 2501 Sur, 64849 Monterrey, NL, Mexico \\ ${ }^{2}$ Escuela de Medicina y Ciencias de la Salud, Tecnológico de Monterrey-Campus Monterrey, \\ Avenida E. Garza Sada 2501 Sur, 64849 Monterrey, NL, Mexico
}

Correspondence should be addressed to Alex Elías-Zúñiga; aelias@itesm.mx

Received 24 March 2013; Accepted 20 May 2013

Academic Editor: Pavel Lejcek

Copyright (C) 2013 Alex Elías-Zúñiga et al. This is an open access article distributed under the Creative Commons Attribution License, which permits unrestricted use, distribution, and reproduction in any medium, provided the original work is properly cited.

\begin{abstract}
This work focuses on the experimental characterization of suture material samples of MonoPlus, Monosyn, polyglycolic acid, polydioxanone $2-0$, polydioxanone $4-0$, poly(glycolide-co-epsilon-caprolactone), nylon, and polypropylene when subjected to cyclic loading and unloading conditions. It is found that all tested suture materials exhibit stress-softening and residual strain effects related to the microstructural material damage upon deformation from the natural, undistorted state of the virgin suture material. To predict experimental observations, a new constitutive material model that takes into account stress-softening and residual strain effects is developed. The basis of this model is the inclusion of a phenomenological nonmonotonous softening function that depends on the strain intensity between loading and unloading cycles. The theory is illustrated by modifying the non-Gaussian averagestretch, full-network model to capture stress-softening and residual strains by using pseudoelasticity concepts. It is shown that results obtained from theoretical simulations compare well with suture material experimental data.
\end{abstract}

\section{Introduction}

Technical advances in the field of surgery have exponentially grown during the last few years. Progressive research, better understanding of the physiopathological processes behind every procedure, more experienced surgeons, and the increasing interest of biomedical companies in developing new products have made possible the excellent results seen in surgery nowadays.

Sutures have remained for many decades a cornerstone for most surgical procedures. Wound closure, vascular and intestinal anastomosis, structures fixation, bleeding control, and tissue approximation are only a few examples from the many uses sutures are given; however, there is no perfect suture for all purposes, and the enormous variables involved in the cicatrization process make this task a difficult one to achieve.

The ideal suture should have certain characteristics that will abet the therapeutic course; it is supposed to have an adequate tensile strength in each phase of the healing process, should be surgeon friendly, induce minimal or no tissue reaction, and must not stimulate infection. Also it should be biologically inert and should be able to have a suitable response to edema and tolerate the different environments within the human body. Since there is no such a product available at present, it is essential to comprehend not only the biological responses to the materials but also understand the precise behavior of each suture in order to decide on the best and most efficient way to take advantage of each particular property of any given suture [1]. Nichols et al. cautioned surgeons about the handling of sutures by surgical instruments since this could result in premature suture failure [2]. They indicated that their rough handling and the usage of clamps and forceps could damage and weaken these.

Therefore, learning the biomechanical performance of sutures will help surgeons not only to determine the appropriate clinical application for each type but also to improve surgical techniques to take advantage of each suture properties [3].

Some of the most important characteristics of a suture regarding its mechanical material properties are related to 
softening and permanent set effects which appear when sutures are subjected to cyclic load, and, thus, the normal stress versus stretch curve shows a reduction on the stress magnitude during the unloading process [4-13]. This stresssoftening effect known as the Mullins effect becomes clinically relevant because initial characteristics exhibited by a suture material immediately after it has been manufactured could change dramatically when stress is applied during the suturing and healing processes [2]. Understanding the material response behavior of suture materials could help surgeons in the selection of the most appropriate suture material.

The aim of this work is to characterize the stress-softening and residual strain effects in suture materials such as polyglycolic acid, polydioxanone, nylon, and polypropylene commonly used in surgical procedures when subjected to loading and unloading cycles by developing a phenomenological nonmonotonous stress-softening hyperelastic material model that depends on the amount of strain [14] and permanent set effects [15].

We have organized this paper as follows. In Section 2, we provide detail of the uniaxial experimental tests performed on suture materials. A brief review of the required equations to describe finite deformations of an incompressible elastic material is introduced in Section 3. In Section 4, we have characterized the experimental data by assuming a nonmonotonous damage softening function and by modifying the Holzapfel et al. constitutive equation to include residual strain effects [15]. Also, we have developed the corresponding stress-stretch constitutive equations by using the nonGaussian average-stretch, full-network model of arbitrarily oriented molecular chains [16]. A comparison of the results corresponding to simulated and experimental data is done in Section 5. Finally, in Section 6, we address some conclusions related to our experimental observations and theoretical predictions of suture materials.

\section{Experimental Work}

2.1. Suture Materials. Eight batches of two suture commercial manufactures were selected to be tested in uniaxial deformation. The absorbable sutures materials tested were MonoPlus, Monosyn, polyglycolic acid, polydioxanone, polydioxanone 4-0, poly(glycolide-co-epsilon-caprolactone) (PGC25 $3-0)$; the nonabsorbable suture materials were nylon and polypropylene. The mean diameter values used to characterize the suture samples were taken from suppliers specifications. The mean values considered here were $0.397 \mathrm{~mm}$ for MonoPlus and Monosyn sutures, $0.334 \mathrm{~mm}$ for polyglycolic acid $2-0,0.377 \mathrm{~mm}$ for polydioxanone $2-0,0.23 \mathrm{~mm}$ for polydioxanone $4-0,0.29 \mathrm{~mm}$ for PGC25 3-0, $0.247 \mathrm{~mm}$ for nylon, and $0.241 \mathrm{~mm}$ for polypropylene.

2.2. Uniaxial Tensile Tests. The experimental tests were performed in two electromechanical universal testing machines. The suture materials identified as MonoPlus, Monosyn, polyglycolic acid 2-0, polydioxanone $2-0$, nylon, and polypropylene were tested in an MTS Insight 2 tensile machine with a maximum cell load capacity of $2.5 \mathrm{kN}$ while the suture materials of polydioxanone 4-0 and PGC25 3-0 were tested

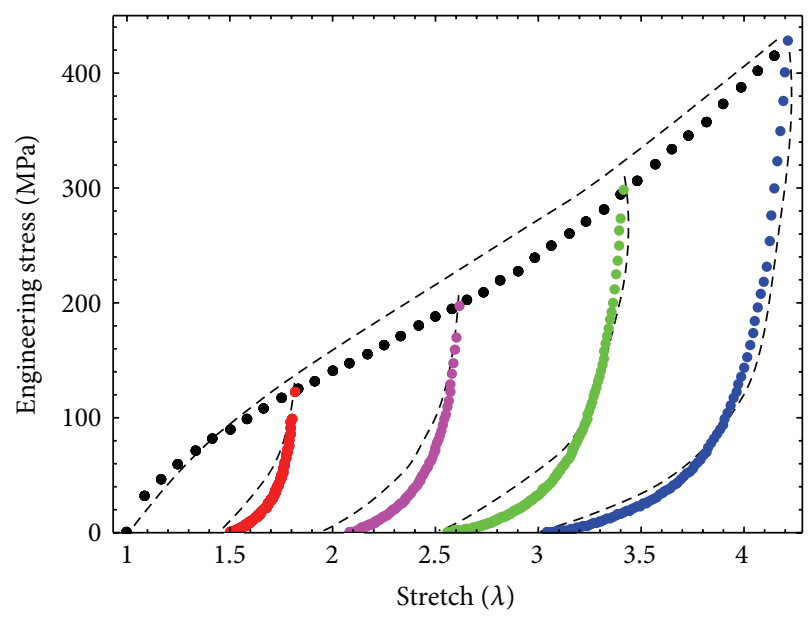

FIGURE 1: Experimental data collected from uniaxial tension cyclic loading-unloading tests for Monosyn sutures.

in an Instron tensile machine Model 3365 with a maximum cell load capacity of $1.6 \mathrm{kN}$. The selected samples length between machine grips was $50 \mathrm{~mm}$. All tests were run at the machine speed of $500 \mathrm{~mm} / \mathrm{min}$ at the average room temperature value of $24^{\circ} \mathrm{C}$. All samples were subjected to cyclic loading-unloading conditions to obtain softening and permanent set effects as shown in Figure 1. Figure 1 illustrates that when the suture material is loaded from its virgin state, unloaded, and then reloaded again, its stress magnitude becomes smaller than the stress magnitude at the same amount of stretch during virgin loading. This reduction in the stress magnitude is known as the Mullins effect $[4,5]$. This softening effect becomes associated with residual strain or permanent set effects which implies that the initial length of the suture sample has increased during the application of the tensile load. Table 1 illustrates the experimental average value of residual strain measured in each suture batch. Notice that suture materials response behavior agrees with Nichols et al. qualitative observation on sutures materials [2]. However, to describe quantitatively the stress-softened and permanent set effects observed on suture materials, a material model must be used. Therefore, to understand the physical relationships behind a material model, we first briefly review some basic knowledge on finite deformations, and, then, we shall derive a material model that is based on non-Gaussian statistical mechanics.

\section{Basic Concepts on Finite Deformations}

We consider the deformation of an incompressible elastic body which in its natural configuration occupies the region $\Omega$. A material particle is considered to be in its undeformed reference configuration of a body at the place $\mathbf{X}=X_{k} \mathbf{e}_{k}$. After a prescribed deformation the body occupies the region $\Omega_{c}$, the current configuration, and the particle at $\mathbf{X}$ moves to the place $\mathbf{x}=\mathbf{x}_{k} \mathbf{e}_{k}$ in a common rectangular Cartesian frame $\varphi=\left\{O ; \mathbf{e}_{k}\right\}$ with origin $O$ and orthonormal basis $\mathbf{e}_{k}$. Thus, the Cauchy-Green deformation tensor $\mathbf{B} \equiv \mathbf{F F}^{T}$ has the form

$$
\mathbf{B}=\lambda_{1}^{2} \mathbf{e}_{11}+\lambda_{2}^{2} \mathbf{e}_{22}+\lambda_{3}^{2} \mathbf{e}_{33},
$$


TABLE 1: Comparison between experimental and predicted residual strain deformations of the selected suture materials.

\begin{tabular}{|c|c|c|c|c|}
\hline Suture material & $\begin{array}{c}\text { Maximum previous stretch } \\
\lambda_{\max }\end{array}$ & Experimental residual strain & Predicted residual strain & Error $(\%)$ \\
\hline \multirow{4}{*}{ MonoPlus } & 2.09363 & 1.6027 & 1.498 & 6.5327 \\
\hline & 3.1709 & 2.2103 & 2.056 & 6.9809 \\
\hline & 4.2483 & 2.7361 & 2.646 & 3.2930 \\
\hline & 5.3309 & 3.3038 & 3.234 & 2.1127 \\
\hline \multirow{4}{*}{ Monosyn } & 1.8165 & 1.4996 & 1.464 & 2.3739 \\
\hline & 2.6165 & 2.0828 & 1.962 & 5.7998 \\
\hline & 3.4165 & 2.5559 & 2.484 & 2.8131 \\
\hline & 4.2165 & 3.0550 & 3.007 & 1.5711 \\
\hline \multirow{4}{*}{$\begin{array}{l}\text { Polyglycolic acid } \\
2-0\end{array}$} & 1.22 & 1.100 & 1.125 & 2.2727 \\
\hline & 1.44 & 1.230 & 1.251 & 1.7070 \\
\hline & 1.66 & 1.380 & 1.381 & 0.0724 \\
\hline & 1.88 & 1.537 & 1.515 & 1.4313 \\
\hline \multirow{4}{*}{ Polydioxanone $2-0$} & 1.5 & 1.200 & 1.277 & 6.4166 \\
\hline & 2.0 & 1.512 & 1.566 & 3.5714 \\
\hline & 2.5 & 1.815 & 1.862 & 3.5895 \\
\hline & 3 & 2.167 & 2.154 & 0.5999 \\
\hline \multirow{4}{*}{ Polydioxanone 4-0 } & 1.2 & 1.051 & 1.051 & 0.7347 \\
\hline & 1.4 & 1.101 & 1.099 & 1.3187 \\
\hline & 1.6 & 1.149 & 1.147 & 0.7879 \\
\hline & 1.8 & 1.194 & 1.198 & 0.1115 \\
\hline \multirow{4}{*}{$\begin{array}{l}\text { Poly(glycolide-co- } \\
\text { epsilon- } \\
\text { caprolactone) } \\
\text { (PGC25 3-0) }\end{array}$} & 1.3 & 1.123 & 1.104 & 1.7270 \\
\hline & 1.6 & 1.216 & 1.206 & 0.8818 \\
\hline & 1.9 & 1.307 & 1.306 & 0.0238 \\
\hline & 2.20 & 1.403 & 1.407 & 0.2851 \\
\hline \multirow{4}{*}{ Nylon } & 1.48 & 1.109 & 1.235 & 11.36 \\
\hline & 1.96 & 1.366 & 1.481 & 8.4187 \\
\hline & 2.44 & 1.67 & 1.744 & 4.4311 \\
\hline & 2.92 & 2.011 & 2.019 & 0.3978 \\
\hline \multirow{4}{*}{ Polypropylene } & 1.4 & 1.154 & 1.197 & 3.72 \\
\hline & 1.8 & 1.361 & 1.4 & 2.8655 \\
\hline & 2.2 & 1.564 & 1.615 & 3.2608 \\
\hline & 2.6 & 1.855 & 1.842 & 0.7008 \\
\hline
\end{tabular}

where $\mathbf{e}_{j k} \equiv \mathbf{e}_{j} \otimes \mathbf{e}_{k}, \mathbf{e}_{i}$ are the associated orthonormal principal directions, $\mathbf{F}$ is the deformation gradient, and $\lambda_{i}$ denote the principal stretches in $\varphi$. Note that the magnitude of the strain intensity at a material point $\mathbf{X}$ denoted by $m$ is defined by $m \equiv \sqrt{\mathbf{B} \cdot \mathbf{B}}=\sqrt{\operatorname{tr} \mathbf{B}^{2}}$, where tr is the trace operation. In the undeformed state $\mathbf{B}=\mathbf{1}$, the identity tensor and $m=\sqrt{3}$; otherwise, $m>\sqrt{3}$ for all isochoric deformations [8]. Also $m \geq \sqrt{3}$ for all $\lambda$, the equality holding when and only when $\lambda=1$, the undeformed state. Recalling that the principal invariants $I_{k}$ of $\mathbf{B}$ are defined by

$$
I_{1}=\operatorname{tr} \mathbf{B}, \quad I_{2}=\frac{1}{2}\left[I_{1}^{2}-\operatorname{tr}\left(\mathbf{B}^{2}\right)\right], \quad I_{3}=\operatorname{det} \mathbf{B},
$$

thus, the magnitude of the strain intensity $m$ is given as

$$
m=\sqrt{I_{1}^{2}-2 I_{2}}
$$

\section{A Nonmonotonous Stress-Softening Material Model}

To characterize stress-softening effects, there exist in the literature many different micromechanical models have been developed to explain material damage mechanisms. See, for instance, the papers of Govindjee and Simo [6], Ogden and Roxburgh [7], Beatty and Krishnaswamy [8], Elías-Zúñiga and Beatty [9], Elías-Zúñiga [11], Diani et al. [12], de Tommasi et al. [13], Holzapfel et al. [15], Johnson and Beatty [17], de Souza Neto et al. [18], Marckmann et al. [19], Dorfmann and Ogden [20], Kazakevičiute-Makovska and Kačianauskas [21], and references cited therein for an overview of the main features of these models.

In this section, we derive the corresponding equations that describe the non-monotonic behavior of suture biocompatible materials subjected to loading and unloading cycles by assuming that the stress-softened material behavior can 
be obtained from the virgin material response constitutive equation. Here, we assume an incompressible and isotropic virgin elastic material whose corresponding time independent Cauchy stress constitutive equation has the form

$$
\mathbf{T}=-p \mathbf{1}+\aleph_{1}\left(I_{1}, I_{2}\right) \mathbf{B}+\aleph_{-1}\left(I_{1}, I_{2}\right) \mathbf{B}^{-1},
$$

in which $\mathbf{T}$ is the Cauchy stress, $p$ is an undetermined pressure, and $\aleph_{\Gamma}=\aleph_{\Gamma}\left(I_{1}, I_{2}\right), \Gamma=1,-1$, denote the virgin material response functions related to the strain energy function $W=\widehat{W}\left(I_{1}, I_{2}\right)$, per unit reference volume, in accordance with

$$
\aleph_{1}=2 W_{1}, \quad \aleph_{-1}=-2 W_{2},
$$

wherein $W_{\alpha} \equiv \partial \widehat{W} / \partial I_{\alpha}[16]$. By using (4), Elías-Zúñiga and Beatty in [9] proposed a damage type model to describe the stress-softened material behavior of the form

$$
\boldsymbol{\tau}=F(m ; M) \mathbf{T}
$$

in which $\boldsymbol{\tau}$ denotes the Cauchy stress in the stress-softened material, $M$ denotes the maximum previous strain at which the material is unloaded from the primary path, and $F(m ; M)$ is an isotropic softening function at the damage level $m_{\max }=$ $M$ on the interval $m \in[\sqrt{3}, M]$. They assumed that this softening function $F(m ; M)$ is a monotone increasing function of the strain intensity that satisfies the conditions

$$
0<F(m ; M)<1, \quad F(M ; M)=1 .
$$

Based on this assumption, Elías-Zúñiga and Beatty proposed the following softening function:

$$
F(m ; M)=e^{-b \sqrt{(M-m)}}
$$

where $b$ is a dimensionless positive material-softening parameter. After substituting (8) into (6), Elías-Zúñiga and Beatty obtained the following stress-softened phenomenological material model:

$$
\boldsymbol{\tau}=e^{-b \sqrt{(M-m)}} \mathbf{T}
$$

Theoretical predictions provided by (9) were computed in Elías-Zúñiga and Beatty [9] and Elías-Zúñiga and Beatty [10] and compared to experimental data for uniaxial extension, pure shear, and equibiaxial deformation states. There, the theoretical predictions showed reasonably good agreement with experimental data not only for the virgin loading path but also for the reloading paths. However, Kazakevičiūtè-Makovska [22] observed that the experimental data when plotted as the normalized stress $\tau / T$ versus the stretch ratio $\lambda / \lambda_{\max }$ showed nonmonotonous behavior with the characteristic $S$ shaped form, and $\lambda_{\max }$ defines the maximum amount of stretch on the primary loading path corresponding to the value at which the unloading starts in a particular deformation cycle. Kazakevičiūtè-Makovska concluded that, because of the variations in shapes of the curves for different deformation cycles, different values of the softening parameters were needed to fit experimental data for a particular choice of the softening function. Moreover, Kazakevičiūtè-Makovska showed that the softening function given by (8) fails in predicting the nonmonotonous behavior exhibited by experimental data collected by Mullins and Tobin [5], Cheng and Chen [23], and Mars and Fatemi [24] at higher stretch values.

On the other hand, de Tommasi and coworkers showed the importance of microscopic inhomogeneity to describe known experimental effects observed in amorphous materials such as the transition from diffuse to localized damage as the distribution properties are varied [25]. In fact, they showed that the monotone stress-stretch loading curve behavior is mainly due to a diffuse damage mechanism. They also considered that amorphous materials may be characterized by unstable strain domain, which gives the possibility of having homogeneous or localized damage with nonmonotone primary loading curve.

To confirm these observations, Elías-Zúñiga and Rodrguez in [14] used the nonmonotonous stress-softening function

$$
F(m ; M)=e^{-b\left[(M-m)(m / M)^{\gamma}\right]^{\alpha}},
$$

where $b$ is a positive softening material parameter and $\alpha$ and $\gamma$ are positive scaling constants chosen to best fit experimental data. They chose the values of $\alpha=1 / 2$ and $\gamma=1$ for the scaling constants and fit the value of the softening parameter $b$ according to the unloading experimental data of the unloading path at which the amount of stretch has the maximum value. Then, they used the equation

$$
\boldsymbol{\tau}=\mathbf{T} e^{-b[(M-m)(m / M)]^{1 / 2}}
$$

to predict the corresponding stress-softened values during the inflation and deflation of rubber balloons.

To account permanent set effects during the material unloading processes, Elías-Zúñiga and Rodrguez [14] modified Holzapfel et al. model [15] and proposed an energy model based on pseudoelastic theory of the form:

$$
W_{s}=W\left(\lambda_{1}, \lambda_{2}, \lambda_{3}\right)+\frac{\mu}{C} \sum_{a=1}^{3}\left[\frac{1}{2}\left(\lambda_{\max a}^{n}-\lambda_{a}^{n}\right)^{2}-C d_{0}\right],
$$

where $W\left(\lambda_{1}, \lambda_{2}, \lambda_{3}\right)$ represents the strain energy function associated with the primary loading path, $\mu$ is the material shear modulus, $C$ is a positive dimensionless material constant, $d_{0}$ is an integration constant, $n$ is a fitting parameter that in general takes the value of $1 / 2, \lambda_{a}$ represents the principal stretches, and $\lambda_{\max a}, a=1,2,3$, are the maximum values of the principal stretches at which unloading begins on the primary loading path.

Here, we use the softening function given by (10) and assume that $W\left(\lambda_{1}, \lambda_{2}, \lambda_{3}\right)$ is provided by the non-Gaussian Arruda-Boyce constitutive equation for an average-stretch, full-network of arbitrarily oriented molecular chains to predict softening and residual strain effects on suture materials.

4.1. A Nonmonotonous Amended Averaged Stretch Material Model. For this material model, it is well known that the 
strain energy per unit volume for the loading path is given by

$$
\begin{aligned}
& W\left(\lambda_{1}, \lambda_{2}, \lambda_{3}\right) \\
& \quad=\mu\left[N_{8}\left(\beta \lambda_{r}+\ln \left(\frac{\beta}{\sinh \beta}\right)\right)-\ln \left(\frac{\beta}{\lambda_{r}}\right)\right]-c_{8},
\end{aligned}
$$

where $\lambda_{r}$ is the relative chain stretch defined by

$$
\lambda_{r}=\frac{\lambda_{\text {chain }}}{\lambda_{L}}
$$

$\lambda_{L}=\sqrt{N_{8}}$ represents the fully extended chain stretch, $N_{8}$ is the chain number of rigid links, each of length $l, \lambda_{\text {chain }}$ is the chain deformation that in the affine deformation is determined by

$$
\lambda_{\text {chain }} \equiv \sqrt{\frac{I_{1}}{3}}
$$

$\beta$ defined by $\beta \equiv \mathscr{L}^{-1}\left(\lambda_{r}\right)$ is the inverse of the Langevin function $\mathscr{L}(\beta)$ which is defined as

$$
\lambda_{r}=\mathscr{L}(\beta) \equiv \operatorname{coth} \beta-\frac{1}{\beta},
$$

and $c_{8}$ is a constant that ensures that the strain energy density vanishes in the undeformed state $[16,26]$. Substitution of (13) into (12) provides the modified non-Gaussian pseudo strain energy per unit volume that accounts for residual strains on the unloading path; that is,

$$
\begin{aligned}
W_{s}= & \mu\left[N_{8}\left(\beta \lambda_{r}+\ln \left(\frac{\beta}{\sinh \beta}\right)\right)-\ln \left(\frac{\beta}{\lambda_{r}}\right)\right] \\
& +\frac{\mu}{C} \sum_{a=1}^{3}\left[\frac{1}{2}\left(\lambda_{\max a}^{n}-\lambda_{a}^{n}\right)^{2}\right]+D,
\end{aligned}
$$

where $D$ is an energy constant.

The Cauchy stress-stretch averaging network model components for the virgin material are obtained by substituting (13) into (4):

$$
T_{k}=-p+\aleph\left(I_{1}\right) \lambda_{k}^{2},
$$

where $\aleph\left(I_{1}\right)$ is a material response function given as

$$
\aleph\left(I_{1}\right) \equiv \frac{\mu}{3 \lambda_{r}}\left[\beta+\frac{1}{N_{8}}\left(\frac{1}{\lambda_{r}}-\frac{1}{\beta\left(1-\lambda_{r}^{2}-2 \lambda_{r} / \beta\right)}\right)\right] .
$$

Eliminating the pressure from (18) gives

$$
T_{j}-T_{k}=\aleph\left(I_{1}\right)\left(\lambda_{j}^{2}-\lambda_{k}^{2}\right),
$$

where $j \neq k=1,2,3$ (no sum). Similarly, the Cauchy stressstretch constitutive equation for a stress-softened material can be obtained by substituting (17) into (4) and by using (5) and (11) yields the following stress-softened components:

$$
\begin{aligned}
\tau_{k}= & {\left[-p+\aleph\left(I_{1}\right) \lambda_{k}^{2}+\frac{\mu \lambda_{k}}{2 C} f_{k}\left(\lambda_{1}, \lambda_{2}, \lambda_{3}\right)\right] } \\
& \times e^{-b \sqrt{(M-m)(m / M)}}, \quad k=1,2,3 \text { (nosum), }
\end{aligned}
$$

where

$$
f_{k}\left(\lambda_{1}, \lambda_{2}, \lambda_{3}\right)=\frac{\partial \sum_{a=1}^{3}\left(\lambda_{\max a}^{n}-\lambda_{a}^{n}\right)^{2}}{\partial \lambda_{k}} .
$$

Then, on elimination of $p$ from (21), it yields

$$
\begin{aligned}
\tau_{j}-\tau_{k}= & {\left[\aleph\left(I_{1}\right)\left(\lambda_{j}^{2}-\lambda_{k}^{2}\right)\right.} \\
& \left.+\frac{\mu}{2 C}\left(\lambda_{j} f_{j}\left(\lambda_{1}, \lambda_{2}, \lambda_{3}\right)-\lambda_{k} f_{k}\left(\lambda_{1}, \lambda_{2}, \lambda_{3}\right)\right)\right] \\
& \times e^{-b \sqrt{(M-m)(m / M)}}
\end{aligned}
$$

where, in general, $j \neq k=1,2,3$ (no sum).

Recalling that, for an incompressible material, the engineering stress $\boldsymbol{\sigma}$ is related to the Cauchy stress by

$$
\sigma=\mathbf{T F}^{-1}
$$

then, the uniaxial engineering stress-stretch relation for an average-stretch, full-network stress-softened material model is obtained by using (22), (23), and (24):

$$
\begin{aligned}
\sigma_{s}=\left[\aleph\left(I_{1}\right)\left(\lambda-\lambda^{-2}\right)\right. \\
+\frac{\mu}{C}\left(-n \lambda^{(n-1)}\left(\lambda_{\max }^{n}-\lambda^{n}\right)\right. \\
\left.\left.\quad+n \lambda^{-(1+n / 2)}\left(\lambda_{\max }^{-n / 2}-\lambda^{-n / 2}\right)\right)\right] \\
\quad \times e^{-b \sqrt{(M-m)(m / M)}} .
\end{aligned}
$$

Here,

$$
m=\sqrt{\lambda^{4}+2 \lambda^{-2}}
$$

and the relative chain stretch which can be obtained from (14) and (15) is given as

$$
\lambda_{r}=\sqrt{\frac{1}{3 N_{8}}\left(\lambda^{2}+2 \lambda^{-1}\right)}
$$

Of course, other material models may be modified by using (10) and our derived pseudo strain energy per unit volume given by (12) to account for a nonmonotonous stress-softened behavior as well as permanent set effects, respectively.

We next examine the degree of accuracy attained by our proposed material model in predicting experimental data of biocompatible suture materials.

\section{Comparison with Suture Experimental Data}

To assess the accuracy of the derived constitutive (25) which includes residual strains and has a nonmonotonous stress softening function that describes Mullins effect, we used the experimental data collected during uniaxial extension test of the aforementioned suture material samples. Notice from 
(25) that only four constitutive material constants and one fitting parameter need to be computed, that is, the shear modulus $\mu$, the chain number of links $N$, the stress softening parameter $b$, the residual strain material constant $C$, and the fitting parameter $n$. However, we have found that in general the value of $n=1$ for the uniaxial stress-softened material model described by (25) provides good fit to the collected experimental data.

We begin with the stress-stretch data for suture samples of MonoPlus and Monosyn materials. Figures 2 and 3 illustrate the predicted engineering stress response curves obtained from (24) and (25). We can see from Figures 2 and 3 that theoretical results are in good agreement with experimental data for the several loading and unloading cycles that exhibit residual strains. The constitutive material constants used to best fit experimental data are $\mu=100 \mathrm{MPa}, N=20, b=0.45$, and $C=0.0065 \mathrm{MPa}$ for MonoPlus sutures and $\mu=92 \mathrm{MPa}$, $N=20, b=0.85$, and $C=0.0045 \mathrm{MPa}$ for Monosyn material. The amount of error attained between experimental and predicted residual strains is shown in Table 1. From Figures 2 and 3 and Table 1, it is concluded that Monosyn sutures tend to soften and have bigger residual strains than MonoPlus sutures. In all figures, the dashed black lines represent theoretical predictions, and the blue solid lines describe experimental data.

Figures 4 through 7 illustrate the stress-stretch curves collected from polyglycolic acid, polydioxanone, polydioxanone 4.0, and poly(glycolide-co-epsilon-caprolactone) (PGC25 3$0)$ suture material samples, respectively. We can see from Figures 4, 5, 6, and 7 that the predicted response stressstretch curves computed from (25) stand in good agreement with experimental data for the several loading and unloading cycles. In these figures, the blue lines represent the experimental collected data, and the dashed black lines represent theoretical results obtained from (24) and (25). The material constants used in the material model are provided by a best fit analysis and these are listed in the figure captions. It is clear from Figures 4-7 that each suture material exhibits different qualitative and quantitative response material behavior. In fact, the amount of softening on the polyglycolic acid 2-0 and polydioxanone 2-0 suture materials is bigger than those of polydioxanone 4.0 and PGC25 3-0.

Finally, Figures 8 and 9 show the stress-stretch curves of the nonabsorbable nylon and polypropylene suture materials. Although the polypropylene sutures are stiffer than the nylon ones, the amount of strength and residual strain are quite similar. Both sutures material experienced stress-softened and permanent set that must be taken into account during suture manipulation to prevent damaging and weakening undesirable effects.

\section{Conclusions}

In this paper, we have examined the material behavior of eight different types of suture materials and found that, when these are subjected to loading and unloading cycles, its stress magnitude becomes lower than that of the virgin material. Furthermore, all tested sutures exhibit residual strains which is related to microstructural material damage upon

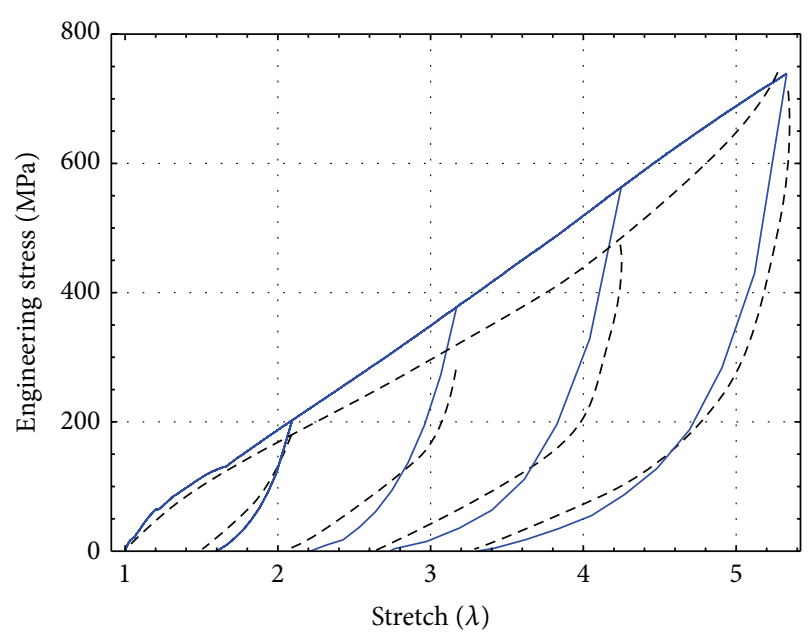

FIGURE 2: Engineering stress-stretch data for MonoPlus sutures compared with theoretical predictions of the nonmonotonous amended average-stretch, full-network model for which $\mu=$ $100 \mathrm{MPa}, N=20, b=0.45$, and $C=0.0065 \mathrm{MPa}$. The dashed black lines represent theoretical predictions, and the blue solid lines describe experimental data.

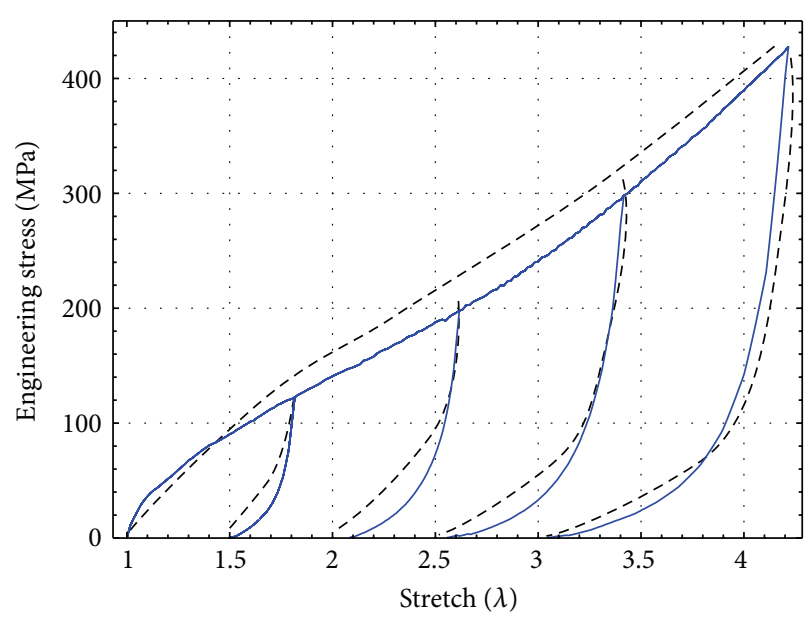

FIGURE 3: Engineering stress-stretch data for Monosyn sutures compared with theoretical predictions of the nonmonotonous amended average-stretch, full-network model for which $\mu=92 \mathrm{MPa}, N=$ $20, b=0.85$, and $C=0.0045 \mathrm{MPa}$. The dashed black lines represent theoretical predictions, and the blue solid lines describe experimental data.

deformation from the natural, undistorted state of the virgin material. To predict the suture materials response behavior observed during uniaxial tension test, we have introduced a new nonmonotonous stress-softened material model that takes into account permanent set effects for the unloading paths as described by the simple constitutive relation (25).

For each suture material, we have compared experimental data with theoretical predictions obtained from (25). In each case, we have determined the corresponding four material constants: the material shear modulus $\mu$, the chain number of rigid links $N$, the material softening parameter $b$, and a positive material constant $C$ that is related to the pseudoelastic 


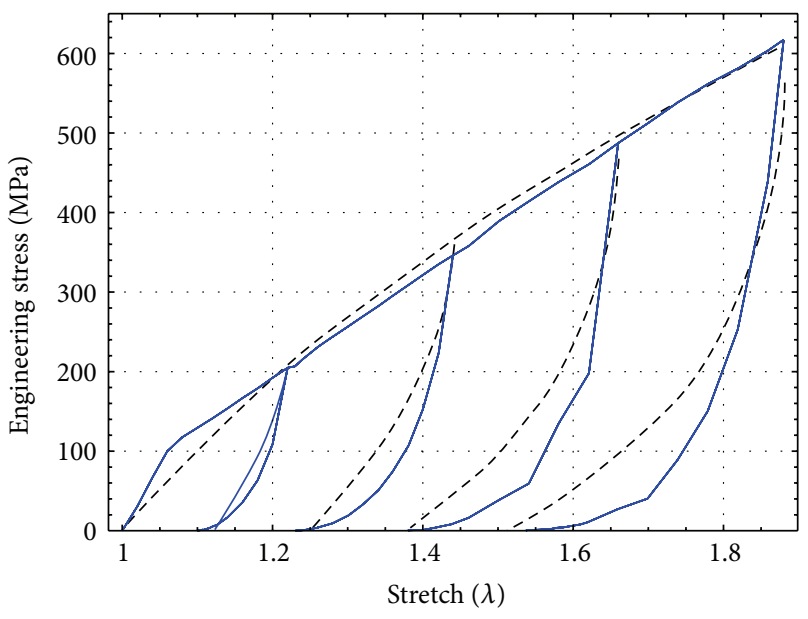

FIgURE 4: Engineering stress-stretch data for polyglycolic acid sutures compared with theoretical predictions of the nonmonotonous amended average-stretch, full-network model for which $\mu=$ $385 \mathrm{MPa}, N=70.5, b=1.3$, and $C=0.001 \mathrm{MPa}$. The dashed black lines represent theoretical predictions, and the blue solid lines describe experimental data.

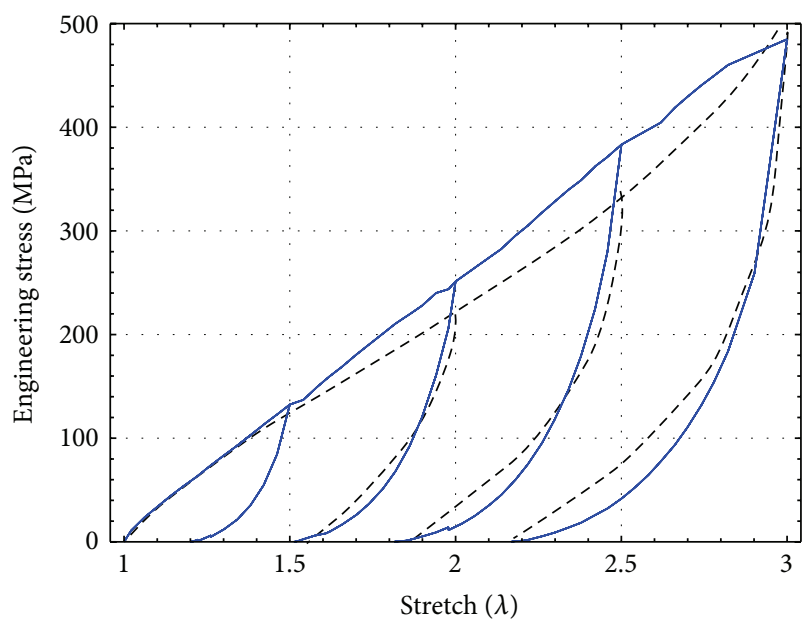

FIGURE 5: Engineering stress-stretch data for polydioxanone 20 compared with theoretical predictions of the nonmonotonous amended average-stretch, full-network model for which $\mu=$ $126 \mathrm{MPa}, N=6, b=0.65$, and $C=0.0035 \mathrm{MPa}$. The dashed black lines represent theoretical predictions, and the blue solid lines describe experimental data.

residual strain energy. Based on the accuracy of our proposed nonmonotonous model to predict experimental data, we can conclude that the extent of damage of suture biocompatible materials can be conveniently determined by considering its softening behavior observed during experimental tests. Nevertheless, there is a variation in the theoretical predictions, as shown in Table 1, that we believe is due to some viscoelastic effects that were not considered in the proposed material model.

Finally, the present study confirms that stress-softening and residual strain effects appear in the suture materials

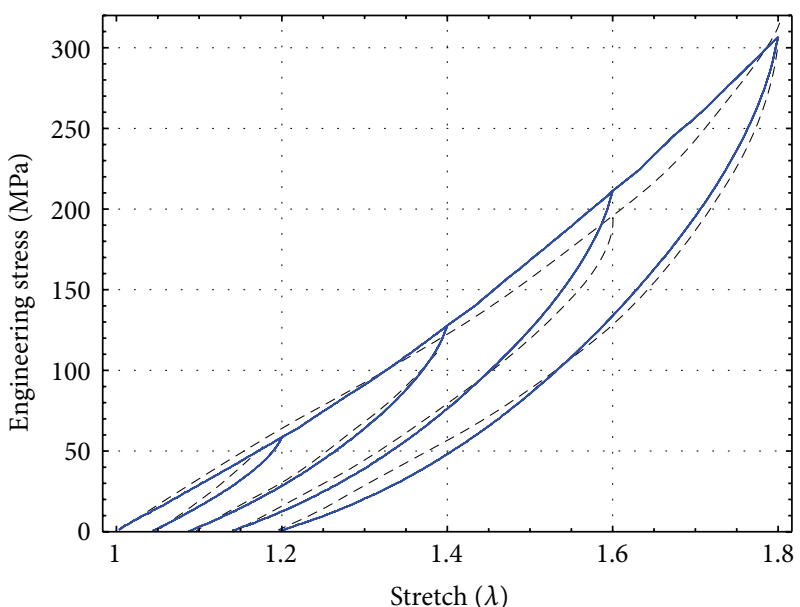

FIgURE 6: Engineering stress-stretch data for polydioxanone 4.0 compared with theoretical predictions of the nonmonotonous amended average-stretch, full-network model for which $\mu=$ $148 \mathrm{MPa}, N=1.95, b=0.445$, and $C=0.0115 \mathrm{MPa}$. The dashed black lines represent theoretical predictions, and the blue solid lines describe experimental data.

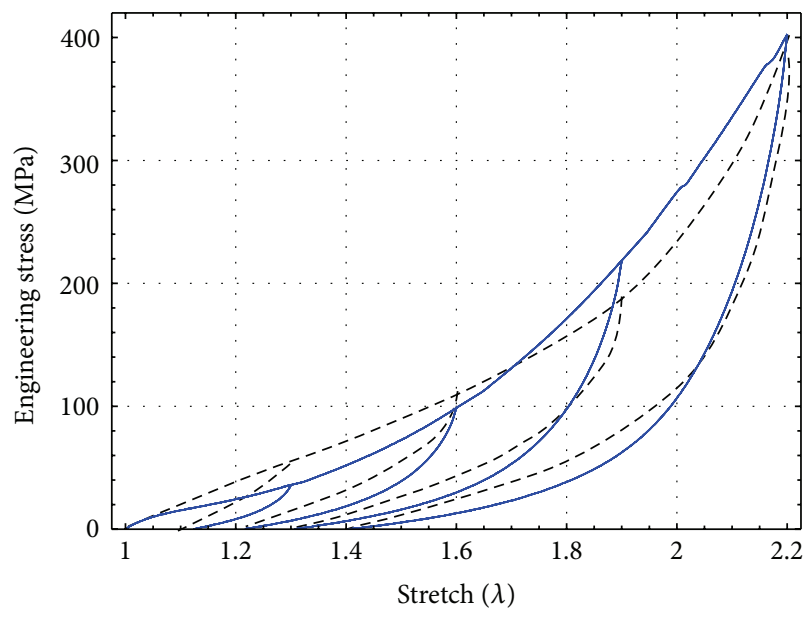

FIgURE 7: Engineering stress-stretch data for PGC25 3-0 sutures compared with theoretical predictions of the nonmonotonous amended average-stretch, full-network model for which $\mu=$ $90 \mathrm{MPa}, N=2.35, b=0.75$, and $C=0.012 \mathrm{MPa}$. The dashed black lines represent theoretical predictions, and the blue solid lines describe experimental data.

tested here. The experimental work of this paper proves that suture materials change dramatically when tensile loads are applied during the suturing and healing processes [2]. We have also found that the aforementioned effects are more evident when in vitro sutures are subjected to cyclic loading conditions. However, the results of this new experimental work will be reported in a subsequent paper.

\section{Conflict of Interests}

The authors declare that they have no conflict of interests. 


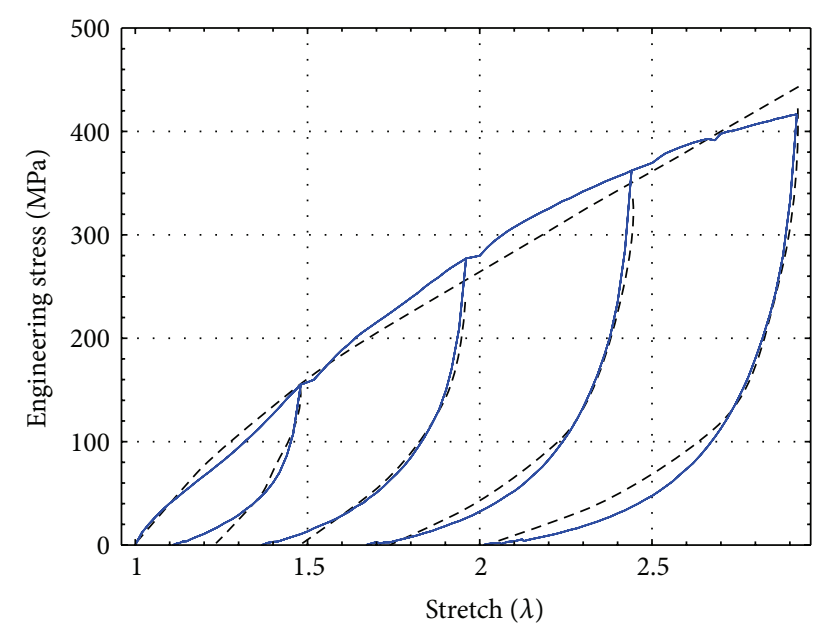

FIGURE 8: Engineering stress-stretch data for nylon sutures compared with theoretical predictions of the nonmonotonous amended average-stretch, full-network model for which $\mu=155 \mathrm{MPa}, N=$ $20.5, b=1$, and $C=0.0035 \mathrm{MPa}$. The dashed black lines represent theoretical predictions, and the blue solid lines describe experimental data.

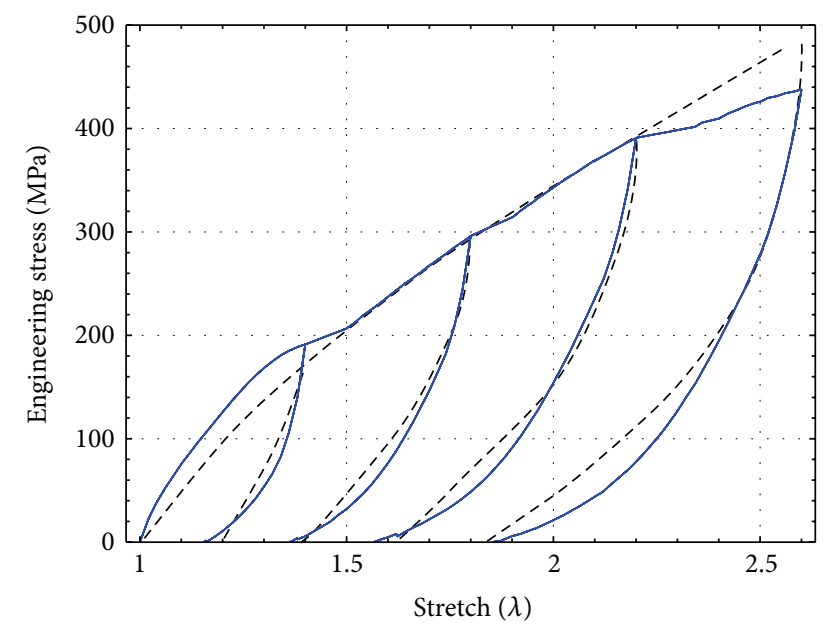

FIGURE 9: Engineering stress-stretch data for polypropylene sutures compared with theoretical predictions of the nonmonotonous amended average-stretch, full-network model for which $\mu=$ $200 \mathrm{MPa}, N=30.5, b=0.65$, and $C=0.00265 \mathrm{MPa}$. The dashed black lines represent theoretical predictions, and the blue solid lines describe experimental data.

\section{Acknowledgments}

This work was funded by Tecnológico de Monterrey-Campus Monterrey, through the Research Chair in Nanomaterials for Medical Devices and Research Chair in Intelligent Machines. Additional support was provided by the project FOMIX Nuevo León M0014-2010-30 \#145045 and from the European Union Seventh Framework Programme (FP7PEOPLE-2009) under the grant agreement IRSES no. 247476.

\section{References}

[1] N. P. Ingle and M. W. King, "Optimizing the tissue anchoring performance of barbed sutures in skin and tendon tissues," Journal of Biomechanics, vol. 43, no. 2, pp. 302-309, 2010.

[2] W. K. Nichols, M. Stanton, D. Silver, and W. F. Keitzer, "Anastomotic aneurysms following lower extremity revascularization," Surgery, vol. 88, no. 3, pp. 366-374, 1980.

[3] M. Poukalova, C. M. Yakacki, R. E. Guldberg et al., "Pullout strength of suture anchors: effect of mechanical properties of trabecular bone," Journal of Biomechanics, vol. 43, no. 6, pp. 1138-1145, 2010.

[4] L. J. Mullins, "Effect of stretching on the properties of rubber," Journal of Rubber Research, vol. 16, pp. 275-289, 1947.

[5] L. Mullins and N. R. Tobin, "Theoretical model for the elastic behavior of filled-reinforced vulcanized rubbers," Journal of Rubber Chemestry and Technology, vol. 30, pp. 555-571, 1957.

[6] S. Govindjee and J. Simo, "A micro-mechanically based continuum damage model for carbon black-filled rubbers incorporating Mullins' effect," Journal of the Mechanics and Physics of Solids, vol. 39, no. 1, pp. 87-112, 1991.

[7] R. W. Ogden and D. G. Roxburgh, "A pseudo-elastic model for the Mullins effect in filled rubber," Proceedings of the Royal Society A: Mathematical, Physical and Engineering Sciences, vol. 455, no. 1988, pp. 2861-2877, 1999.

[8] M. F. Beatty and S. Krishnaswamy, "Theory of stress-softening in incompressible isotropic materials," Journal of the Mechanics and Physics of Solids, vol. 48, no. 9, pp. 1931-1965, 2000.

[9] A. Elías-Zúñiga and M. F. Beatty, "A new phenomenological model for stress-softening in elastomers," Zeitschrift fur Angewandte Mathematik und Physik, vol. 53, no. 5, pp. 794-814, 2002.

[10] A. Elías-Zúñiga and M. F. Beatty, "Stress-softening effects in the transverse vibration of a non-Gaussian rubber string," Meccanica, vol. 38, no. 4, pp. 419-433, 2003.

[11] A. Elías-Zúñiga, "A phenomenological energy-based model to characterize stress-softening effect in elastomers," Polymer, vol. 46, no. 10, pp. 3496-3506, 2005.

[12] J. Diani, M. Brieu, and J. M. Vacherand, "A damage directional constitutive model for Mullins effect with permanent set and induced anisotropy," European Journal of Mechanics, A/Solids, vol. 25, no. 3, pp. 483-496, 2006.

[13] D. de Tommasi, G. Puglisi, and G. Saccomandi, "A micromechanics-based model for the Mullins effect," Journal of Rheology, vol. 50, no. 4, pp. 495-512, 2006.

[14] A. Elías-Zúñiga and C. A. Rodríguez, "A non-monotonous damage function to characterize stress-softening effects with permanent set during inflation and deflation of rubber balloons," International Journal of Engineering Science, vol. 48, no. 12, pp. 1937-1943, 2010.

[15] G. A. Holzapfel, M. Stadler, and R. W. Ogden, "Aspects of stress softening in filled rubbers incorporating residual strains," in Proceedings of the first European Conference on Constitutive Models for Rubber, A. Dorfmann and A. Muhr, Eds., pp. 189193, Rotterdam, The Netherlands, 1999.

[16] M. F. Beatty, "An average-stretch full-network model for rubber elasticity," Journal of Elasticity, vol. 70, no. 1-3, pp. 65-86, 2003.

[17] M. A. Johnson and M. F. Beatty, "The mullins effect in uniaxial extension and its influence on the transverse vibration of a rubber string," Continuum Mechanics and Thermodynamics, vol. 5, no. 2, pp. 83-115, 1993. 
[18] E. A. de Souza Neto, D. Perić, and D. R. J. Owen, "A phenomenological three-dimensional rate-idependent continuum damage model for highly filled polymers: formulation and computational aspects," Journal of the Mechanics and Physics of Solids, vol. 42, no. 10, pp. 1533-1550, 1994.

[19] G. Marckmann, E. Verron, L. Gornet, G. Chagnon, P. Charrier, and P. Fort, "A theory of network alteration for the Mullins effect," Journal of the Mechanics and Physics of Solids, vol. 50, no. 9, pp. 2011-2028, 2002.

[20] A. Dorfmann and R. W. Ogden, "A constitutive model for the Mullins effect with permanent set in particle-reinforced rubber," International Journal of Solids and Structures, vol. 41, no. 7, pp. 1855-1878, 2004.

[21] R. Kazakevičiute-Makovska and R. Kačianauskas, "Modelling of stress softening in elastomeric materials: foundations of simple theories," Mechanics Research Communications, vol. 31, no. 4, pp. 395-403, 2004.

[22] R. Kazakevičiūtè-Makovska, "Experimentally determined properties of softening functions in pseudo-elastic models of the Mullins effect," International Journal of Solids and Structures, vol. 44, no. 11-12, pp. 4145-4157, 2007.

[23] M. Cheng and W. Chen, "Experimental investigation of the stress-stretch behavior of EPDM rubber with loading rate effects," International Journal of Solids and Structures, vol. 40, no. 18, pp. 4749-4768, 2003.

[24] W. V. Mars and A. Fatemi, "Observations of the constitutive response and characterization of filled natural rubber under monotonic and cyclic multiaxial stress states," Journal of Engineering Materials and Technology, vol. 126, no. 1, pp. 19-28, 2004.

[25] D. de Tommasi, G. Puglisi, and G. Saccomandi, "Localized versus diffuse damage in amorphous materials," Physical Review Letters, vol. 100, no. 8, Article ID 085502, pp. 1-4, 2008.

[26] A. Elías-Zúñiga and M. F. Beatty, "Constitutive equations for amended non-Gaussian network models of rubber elasticity," International Journal of Engineering Science, vol. 40, no. 20, pp. 2265-2294, 2002. 

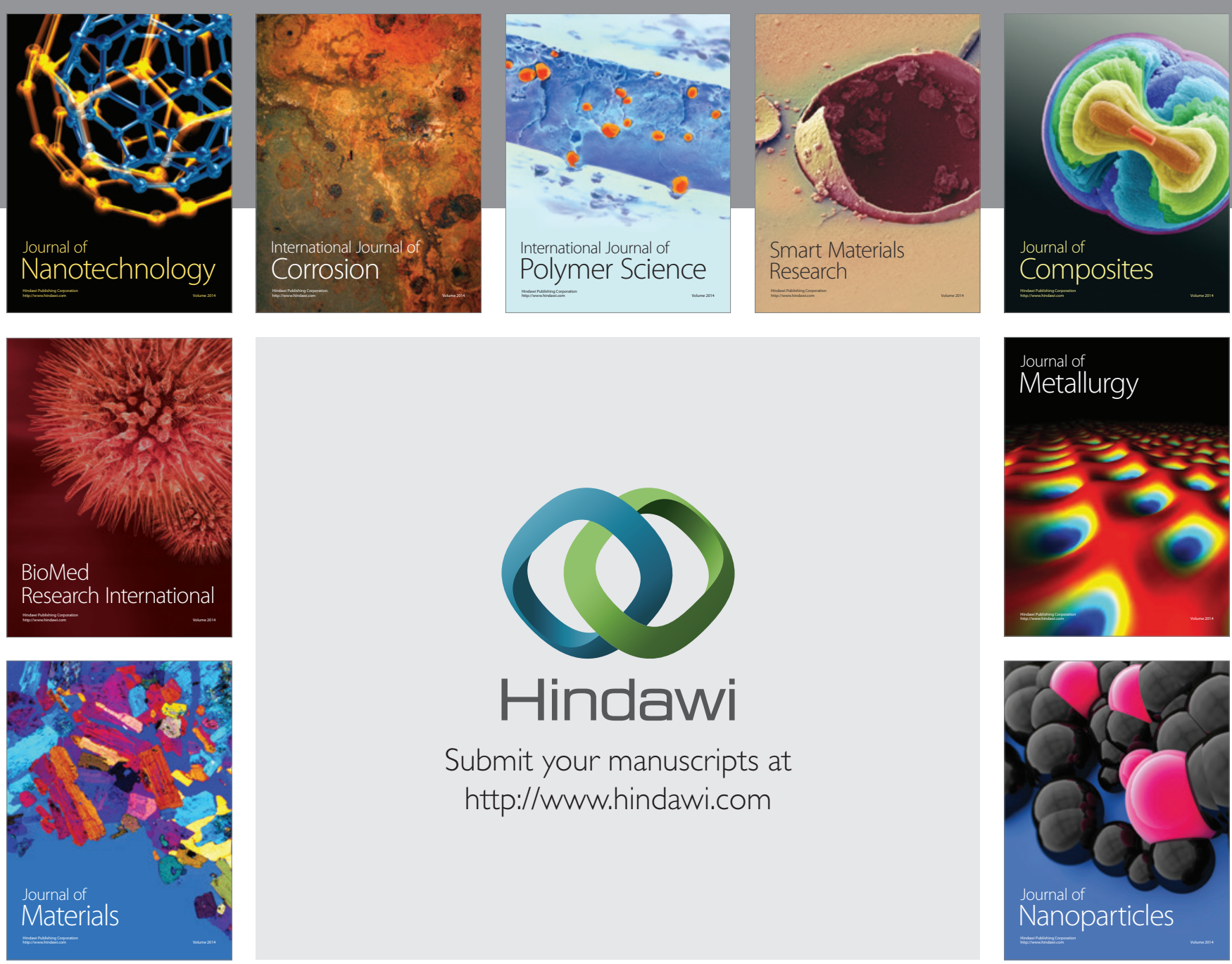

Submit your manuscripts at http://www.hindawi.com
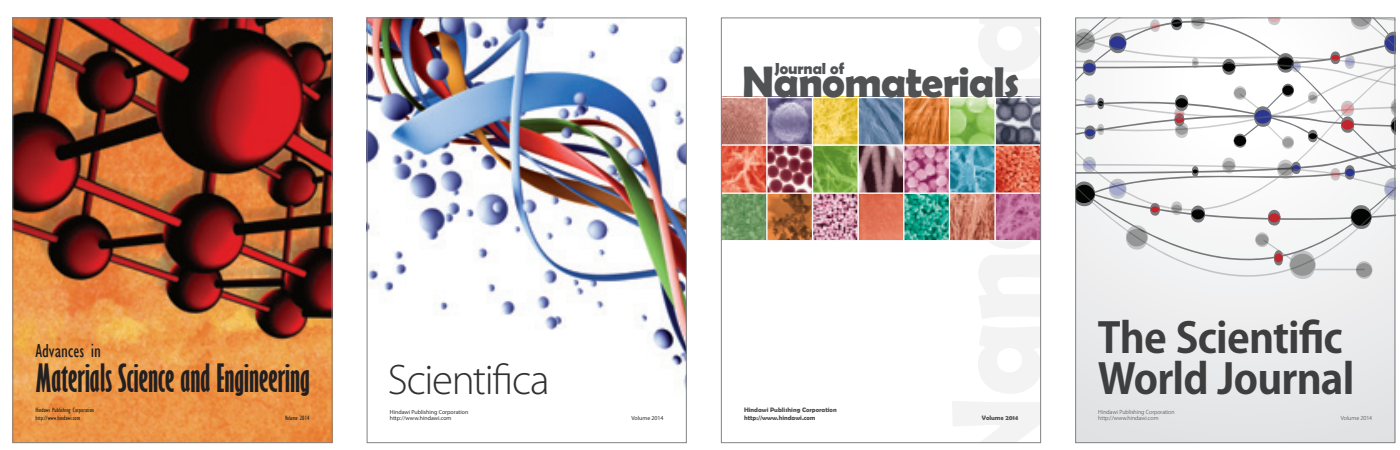

\section{The Scientific World Journal}
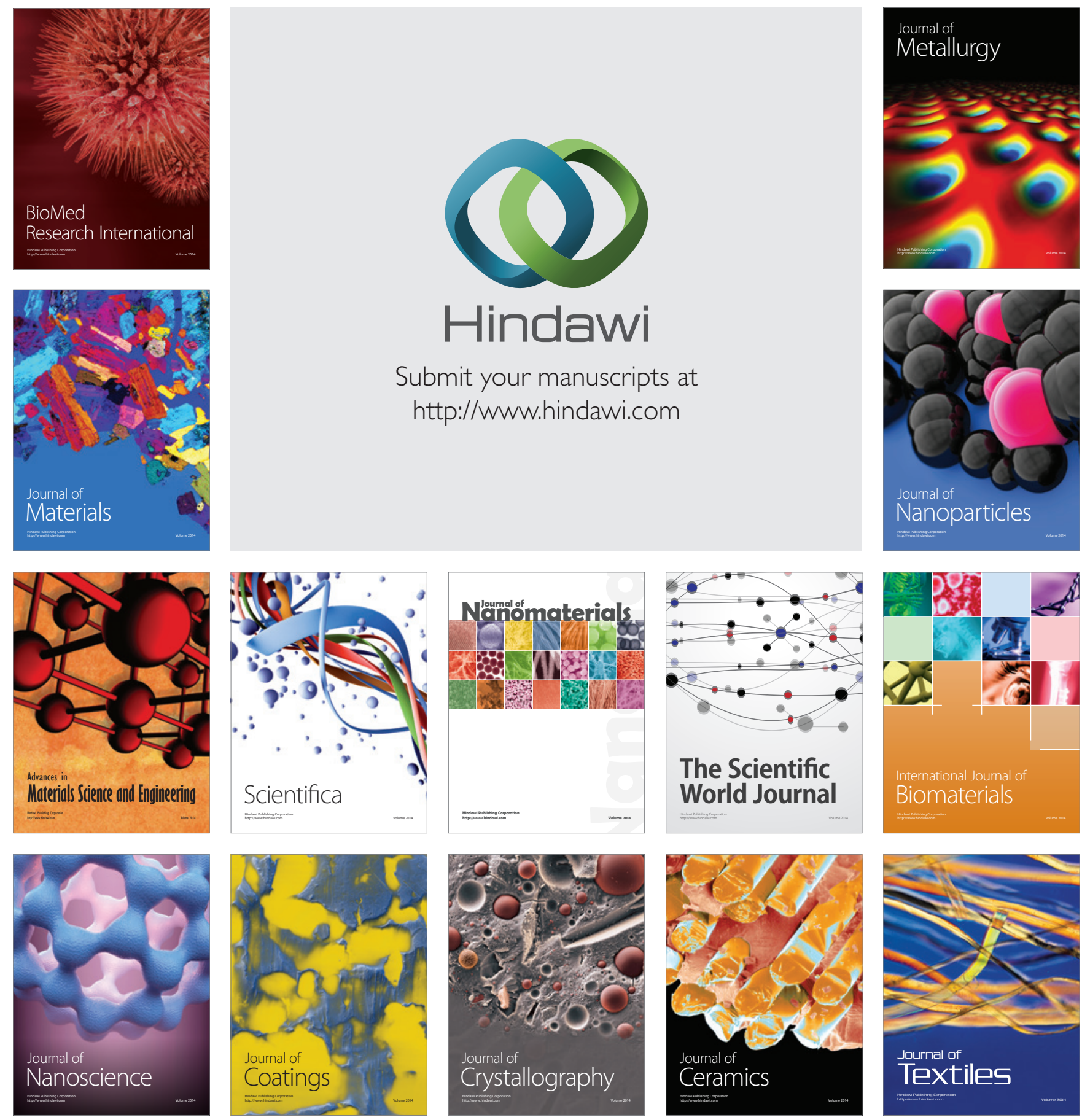